\title{
Der Notarzt - ein wichtiger Spezialist im schweizerischen Rettungswesen
}

Stefan Müller ${ }^{a}$,

Wolfgang Ummenhofer ${ }^{b}$

a Dr. med., Ärztlicher Leiter Schutz \& Rettung Zürich

b Prof. Dr. med.

Departement Anästhesie, Universitätsspital Basel

Wir bedanken uns bei Bruno Durrer und Heinz Zimmermann für die kritische Durchsicht des Manuskripts.

Korrespondenz: Prof. Dr. med. Wolfgang Ummenhofer Universitätsspital Basel Departement Anästhesie CH-4031 Basel

ummenhoferw[at]uhbs.ch
Die Plattform Rettungswesen der FMH hat im Thesenpapier zum Rettungswesen in der Schweiz bereits 1996 darauf hingewiesen, dass die präklinische Notfall- und Rettungsmedizin speziell ausgebildete Ärzte, sogenannte Notärzte, braucht. Dies gilt noch heute, und das 2010 aktualisierte Leitbild zum Rettungswesen zeigt Ansätze für regional sinnvolle Lösungen auf. Die FMH engagiert sich, dass das «duale System» der Notfallversorgung durch Rettungssanitäter einerseits und Notärzte bzw. Dienstärzte andererseits in der Praxis umgesetzt wird.

Notärzte müssen deshalb gemäss kantonalen Einsatzkonzepten in anerkannten Institutionen und Rettungsorganisationen so integriert werden, dass sie jederzeit ausrücken können. Dafür sind «stationäre» und «Rendez-vous»-Systeme möglich. Im stationären System rückt der Notarzt als Besatzungsmitglied des Rettungswagens (RTW) aus. Im Rendezvous-System fährt der Notarzt unabhängig vom RTW mit einem Notarzteinsatz-Fahrzeug (NEF) zum Notfallort und trifft sich dort mit dem RTW-Team. Insbesondere das Rendez-vous-System erlaubt es, auch niedergelassene Notärzte einzubinden. Beide Systeme haben Vor- und Nachteile und müssen den regionalen Gegebenheiten angepasst werden. Bei spitalgebundenen Rettungsdiensten in der Peripherie kann das stationäre System bei langen Anfahrtszeiten von Vorteil sein. Der Notarzt kann hier aber auch alternativ durch die Flugrettung an den Notfallort gebracht werden. In Ballungsgebieten mit häufigen Paralleleinsätzen, wo Notärzte für mehrere Rettungsdienste zur Verfügung stehen, erweist sich das Rendez-vous-System als flexibler.

Sanitätsnotrufzentralen (SNZ) müssen bei vital bedrohten Notfallpatienten prinzipiell Rettungssanitäter und Notarzt aufbieten. Die Notarztindikation kann aber durch geographische Gegebenheiten modifiziert werden. So ist es denkbar, dass bei kurzen Transportzeiten akute Koronarpatienten primär von Rettungssanitätern versorgt werden und der Notarzt nur für instabile Verläufe oder eine Reanimation hinzugezogen bzw. nachgefordert wird. In der Peripherie mit langen Transportzeiten ist es dagegen sinnvoll, den Notarzt aufzubieten. Die jeweils definierten Notarztindikationen sind dann für die SNZ verbindlich. Entsprechend müssen sich auch alle Notarzt-Trägerinstitutionen so organisieren, dass ein von der SNZ disponierter Einsatz sofort übernommen werden kann; bei spitalgebundenen Systemen müssen die Dienstpläne deshalb zwingend auf die sofortige Abkömmlichkeit des diensthabenden Notarztes ausgerichtet sein.

Der Interverband Rettungswesen (IVR) ist verantwortlich für die Zertifizierung und das Qualitätsmanagement der Rettungsorganisationen und prüft deshalb die Einhaltung der Notarzt-Einsatzindikationen. Dabei kann es Abweichungen von starren Indikationsvorgaben geben: bei akuter Verschlechterung im Rettungswagen kurz vor Spitalankunft kann die Weiterfahrt ohne Notarzt sinnvoll sein. In solchen Fällen muss das Nichteinhalten der Notarztindikation aber intern gemeldet, besprochen und für den IVR nachvollziehbar dokumentiert sein.

Das Schweizerische Institut für ärztliche Weiterund Fortbildung (SIWF) der FMH regelt die Weiterbildung zum Notarzt. Mit der minimalen Voraussetzung einer dreijährigen klinischen Weiterbildung wird sichergestellt, dass nur erfahrene Kollegen für diesen Dienst eingeteilt werden. Weiter sind der erfolgreiche Besuch des Notarztkurses und die Kurse «Advanced Cardiac Life Support» und «Advanced Pediatric Life Support» vorgeschrieben. Zudem müssen je dreimonatige Tätigkeiten auf einer Notfallund auf einer Intensivstation nachgewiesen werden. Nach 50 supervisierten und dokumentierten Notarzteinsätzen der Dignität NACA 4 oder höher an einer anerkannten Weiterbildungsstätte vergibt die Schweizerische Gesellschaft für Notfall- und Rettungsmedizin (SGNOR) den Fähigkeitsausweis für Präklinische Notfallmedizin (Notarzt).

Die Plattform Rettungswesen der FMH entwickelt als Forum der ärztlichen Fachgesellschaften Leitlinien für eine einheitliche und verbindliche präklinische Notfallversorgung, auch bei Grossereignissen. Die FMH setzt sich dafür ein, dass die Notfallversorgung gemeinsam durch Notärzte bzw. speziell ausgebildete Hausärzte einerseits und Rettungssanitäter andererseits gewährleistet wird. Im Sommer 2010 hat die Plattform Rettungswesen ihr Thesenpapier zum Rettungswesen in der Schweiz aus dem Jahr 1996 aktualisiert. (Schweizerische Ärztezeitung Nr. 33/ 2010, S. 1215 f. und www.fmh.ch $\rightarrow$ SERVICES $\rightarrow$ Plattform Rettungswesen). Periodisch soll nun in der Ärztezeitung auf die einzelnen der acht Punkte des neuen Leitbilds näher eingegangen werden. Der Notarzt - ein wichtiger Spezialist im schweizerischen Rettungswesen. 
Die hochgestellten Notarztanforderungen garantieren, dass vitalbedrohte Patienten kompetent versorgt und transportiert werden. Auch für die Rettungssanitäter ist es wichtig, dass der Notarzt im Team eine erhöhte Versorgungsqualität garantiert. Daher setzt sich die FMH dafür ein, dass in einer Jacke mit der Aufschrift «Notarzt» auch ein Notarzt ausrückt und dass alle eingesetzten Notärzte einen gültigen Fähigkeitsausweis haben; an den anerkannten Weiterbildungsstätten sollen Notärzte in Weiterbildung unter enger Supervision eingesetzt werden. Inhaber ausländischer Zertifikate können bei Äquivalenz ihrer Ausbildung den Fähigkeitsausweis bei der SGNOR beantragen.

Der erhebliche personelle und logistische Aufwand für die Notarztweiterbildung muss im Notfallalltag auch umgesetzt werden können. Leider ist an vielen Spitälern die Notarzttätigkeit nur auf Stufe Assistenzarzt vorgesehen. Häufig können Kaderärzte durch den spitalinternen Leistungsauftrag nicht mehr persönlich am Notarztdienst teilnehmen. Dieser Systemfehler verhindert, dass Notfallpatienten von Wissen, Können und Erfahrung der Kader-Notärzte profitieren können. Dass die präklinische Notfallmedizin nur in einer bestimmten Ausbildungsstufe ausgeübt wird, erklärt wahrscheinlich auch die eher fehlende Forschungsaktivität im Bereich der Notfall- und Rettungsmedizin.

In der «Leitbild-spezifischen Weiterbildung» fokussiert die SGNOR verstärkt auf eine interdisziplinäre Ausrichtung des präklinischen Fähigkeitsausweises: Zusätzlich zu einem Jahr Anästhesie muss die dreijährige klinische Weiterbildung entweder ein Jahr Innere Medizin, Pädiatrie oder ein operatives Fachgebiet beinhalten. Im Weiterbildungsjahr Anästhesie sollen Nicht-Fachanästhesisten mit den «Basic skills» des Atemwegsmanagements und den grundlegenden Techniken des Venenzugangs und der Notfallpharmakologie vertraut werden. Die Schweizerische Gesellschaft für Anästhesie und Reanimation (SGAR) hat für dieses «Fremdjahr» ein spezifisches Curriculum erstellt, womit die Grundlagen der
Anästhesie und Reanimation für die Notfallmedizin vermittelt werden.

Im Gegensatz zur rein «paramedizinischen» Versorgung in angelsächsischen Ländern sollen in der kleinen und dichtbesiedelten Schweiz Not- und Dienstärzte in der präklinischen Notfall- und Rettungsmedizin zum Einsatz kommen. Diese Ärzte sollen aber nicht nur eine Supervisions- oder Leitungsfunktion einnehmen; sie müssen bei Bedarf unmittelbar die Behandlung von vital gefährdeten Notfallpatienten optimieren. Eine solche Chance ärztlicher Beteiligung verpflichtet aber auch zu hoher Ausbildungs- und Ausrüstungsqualität sowie zur Entwicklung und Einhaltung nachhaltiger Einsatzkonzepte.

\section{Kernsätze:}

- Notärzte sind Ärzte, die gemäss den Anforderungen von SIWF und SGNOR in der präklinischen Notfall- und Rettungsmedizin ausgebildet sind und den Fähigkeitsausweis erworben haben. Die Aus- und Weiterbildung der Notärzte muss durch erfahrene Notärzte garantiert werden.

- Abhängig von den örtlichen Gegebenheiten steht das Rendez-vous- oder das stationäre Modell zur Verfügung.

- Notarztindikationen sind durch den Interverband für Rettungswesen (IVR) vorgegeben; sie können den örtlichen Gegebenheiten angepasst werden, müssen dann aber auch begründet werden.

- Die gute Zusammenarbeit zwischen Rettungssanitäter und Notarzt erhöht die Sicherheit der Patienten.

- Eine verstärkte Interdisziplinarität in der Aus- und Weiterbildung bildet das Patientenkollektiv am besten ab und wirkt der Segregation ärztlicher Organisationen entgegen. 